\title{
SIMULADOR CARDIOVASCULAR PARA ENSAYO DE ROBOTS DE NAVEGACIÓN AUTÓNOMA
}

José Emilio Traver ${ }^{1}$, Juan Francisco Ortega Morán ${ }^{2}$, Inés Tejado ${ }^{1}$, J. Blas Pagador ${ }^{2}$,

Fei Sun ${ }^{2}$, Raquel Pérez-Aloe ${ }^{1}$, Blas M. Vinagre ${ }^{1}$, F. Miguel Sánchez Margallo ${ }^{2}$

${ }^{1}$ Escuela de Ingenierías Industriales, Universidad de Extremadura, Avda. de Elvas, s/n, 06006 Badajoz, e-mail: \{jetraver,itejbal,raquel,bvinagre\}@unex.es

${ }^{2}$ Centro de Cirugía de Mínima Invasión Jesús Usón, Ctra. N-521 km 41, 10004 Cáceres, e-mail: \{jfortega,jbpagador,feisun\}@ccmijesususon.com

\section{Resumen}

Este artículo presenta un modelo de simulación del sistema cardiovascular en el entorno de Matlab/Simulink, más concretamente de la zona de mayor riesgo cardiovascular, la arteria carótida. Está basado en un modelo eléctrico del sistema que describe la dinámica de contracción del corazón, así como su carácter cíclico y autónomo. Como primer paso, este modelo se generaliza para contemplar también la dinámica de la arteria carótida izquierda. A partir de él, y haciendo una serie de equivalencias entre dominios, se obtiene un modelo hidráulico que emula el comportamiento del sistema cardiovascular en esa zona y que, a diferencia del anterior, no presenta carácter autónomo. Para el diseño del control, se hace uso de la estrategia de linealización por realimentación. Se incluyen simulaciones, tanto del modelo eléctrico completo como del hidráulico propuesto, para demostrar el correcto funcionamiento del simulador desarrollado. El objetivo final de este trabajo es la construcción de una plataforma de ensayo para robots nadadores tipo flagelo eucariótico y bacteriano de pequeñas dimensiones a partir del modelo hidráulico desarrollado que permita emular las condiciones en las que se encontrarían estos robots navegando por el sistema circulatorio humano.

Palabras clave: Sistema cardiovascular, modelo eléctrico, modelo hidráulico, linealización por realimentación, Simulink, plataforma de ensayo, robots de pequeñas dimensiones.

\section{INTRODUCCIÓN}

Las enfermedades cardiovasculares se consideran como la primera causa de defunción a nivel global, y la tendencia del número de incidencias de este tipo es creciente [11]. Esta circunstancia pone de manifiesto la gravedad de la situación, la necesidad de su estudio y el desarrollo de medios que permitan evaluar y mejorar su tratamiento. Como consecuencia de esta situación, son numerosos y conocidos los esfuerzos combinados de la comunidad médica e ingenieril [1] para el desa- rrollo de modelos y simuladores, conocidos como lazos de circulación Mock (MCLs, del término en inglés). Estos modelos permiten un ahorro en coste y tiempo, además de servir de herramientas para el diseño, evaluación y mejora del funcionamiento de dispositivos de asistencia ventricular $[13,6]$, prótesis de válvulas cardíacas [3] o emuladores del sistema circulatorio para aprendizaje y evaluación de herramientas de cateterismo.

$\mathrm{El}$ sistema cardiovascular (SCV) es un sistema relativamente complejo y son numerosos los modelos desarrollados para su investigación, así como las perspectivas desde las que han sido abordados. Sin embargo, a pesar de la abundante información que es posible encontrar en la literatura respecto al modelado del SCV [13, 6, 4, 18], ésta es escasa en lo que se refiere a tratar los problemas originados por la enfermedad de arteriosclerosis, concretamente en el oclusión (estenosis) de las arterias debido su engrosamiento [10]. Esto se encuentra motivado principalmente por tres razones: 1) las herramientas actuales se encuentran enfocadas principalmente a reducir los síntomas o eliminar las dolencias derivadas de la enfermedad, de forma concreta y localizada, sin la eliminación de los factores que la originan, como es el caso del uso de dispositivos de estabilización (stent) o cirugía de bypass; 2) recientes estudios avalan una mayor eficacia de los tratamientos farmacéuticos frente a las técnicas de tratamientos invasivos, además de ofrecer un tratamiento sistémico [10]; y 3) la ausencia de dispositivos capaces de tratar la enfermedad de forma sistémica. Sin embargo, a partir de la última década los avances tecnológicos han permitido el estudio de dispositivos dentro del campo de la robótica de pequeña escala, como aplicación de la nano y microrrobótica al ámbito médico [9].

En este contexto, el presente trabajo pretende recopilar los fundamentos teóricos necesarios para el desarrollo de un simulador hidráulico que permita emular la dinámica del SCV. Para ello, se partirá de un modelo eléctrico que describe la dinámica de contracción del corazón de acuerdo con la ley de Frank-Starling, así como su carácter cíclico y autónomo. Como primer paso, este modelo se generalizará para incluir también la dinámica de la 
arteria carótida izquierda, que constituye la región con mayor recurrencia en problemas cardiovasculares, y más concretamente, en la formación de placas de ateroma. Basado en éste, se desarrollará un modelo hidráulico que, a diferencia del anterior, no presentará carácter autónomo y necesitará ser controlado para emular la dinámica cardiovascular. Se utilizará el método de linealización por realimentación para el diseño del control. Con ello se logrará independizar la dinámica del sistema de la caracterización de la ley de Frank-Starling, pudiendo así emular el comportamiento descrito por el modelo eléctrico. Ambos modelos, tanto el eléctrico completo como el hidráulico, se implementarán en el entorno Matlab/Simulink usando la herramienta Simscape para validarlos.

El objetivo final de este trabajo es la construcción de una plataforma experimental a partir del modelo hidráulico desarrollado para el ensayo de robots de pequeñas dimensiones, concretamente robots nadadores tipo flagelo eucariótico y bacteriano. Se pretende, pues, emular las condiciones en las que se encontrarían estos robots navegando por el sistema circulatorio humano. Cabe resaltar que en la literatura es posible encontrar modelos del SCV, principalmente eléctricos, de mayor complejidad que el aquí considerado. Sin embargo, no contemplan las arterias centrales, al contrario que los aquí considerados, y están enfocados a otros estudios.

El resto del documento está estructurado como sigue. La sección 2 resume el funcionamiento del SCV. La sección 3 describe y analiza un modelo eléctrico equivalente del SCV, lo extiende para incluir la dinámica de la arteria carótida izquierda, y comprueba su validez con simulaciones en Matlab. Basado en este modelo, la sección 4 presenta un modelo hidráulico que emula la dinámica del SCV. Finalmente, la sección 5 resume las principales conclusiones del trabajo.

\section{DESCRIPCIÓN DEL SISTEMA CARDIOVASCULAR}

Con el objeto de poder realizar una correcta descripción y modelado del SCV, a continuación se resume el funcionamiento del órgano principal, así como la importancia de la red de distribución y su hemodinámica. Conviene tener presente en todo momento que se está describiendo un organismo vivo, y por lo tanto, es importante no olvidar la variabilidad de sus parámetros.

En términos sencillos, es posible describir el SCV como una red de distribución (vasos sanguíneos) que abastece de fluido (sangre) a una población por medio de una bomba (corazón). El corazón ge- nera la presión necesaria para bombear la sangre a través del conjunto de vasos sanguíneos que recorren las células del cuerpo. En el flujo de sangre se distinguen dos etapas: 1) circulación sistémica o general, que es la encargada del transporte de sustancias y oxígeno, y 2) circulación pulmonar, responsable de la oxigenación de la sangre [8].

El corazón actúa como una bomba para cada circulación. Se encuentra constituido por una doble cámara aurícula-ventrículo, donde las aurículas actúan como cámara de precarga y los ventrículos hacen la función de bomba. Así mismo, presenta válvulas unidireccionales que impiden el reflujo entre cámaras (válvulas auriculoventriculares) y a la salida de los ventrículos (válvulas semilunares). La capacidad del corazón para generar el gradiente de presión necesario reside en su capacidad de contracción y la sucesión de fenómenos eléctricos y mecánicos ocurridos durante un latido, conocidos como ciclo cardíaco [8].

El ciclo cardíaco se divide en dos etapas claramente diferenciadas que ocurren de forma alternada: diástole (periodo de relajación) y sístole (periodo de contracción). Las manifestaciones físicas correspondientes a cada una de ellas se ilustran en la Figura 1, donde puede observarse la presión y los cambios de volumen registrados en la aurícula y ventrículo izquierdo (VI) para un conjunto de ocho etapas [8].

Esta dinámica no se preserva durante todo el sistema circulatorio, debido a su diversidad en morfologías, diámetros y composiciones. Por lo tanto, la oposición al flujo de sangre también es variable. La resistencia vascular, entendida como la fricción que experimenta la sangre con las paredes, depende principalmente de dos factores: la longitud y

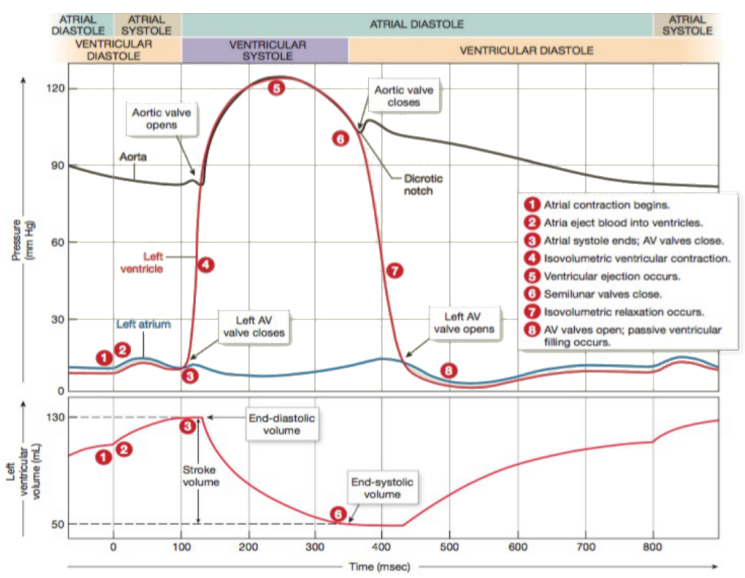

Figura 1: Relación de presiones en el VI durante el ciclo cardíaco para una frecuencia cardíaca de 75 latidos por minuto (lpm). Imagen extraída de [8]. 
el diámetro del vaso. Dicha resistencia es menor en las zonas vasculares (capilares) debido a que su sección total es superior a las zonas adyacentes al corazón (arterias y venas).

Respecto a la presión carótida, presenta ligeras diferencias en comparación con la ilustrada en la Figura 1: 1) existe un desfase de tiempo respecto a la presión sistólica, 2) la presión experimenta un ligero aumento a raíz de las ondas de presión reflejadas, y 3) la onda diacrótica se intensifica [10]. En cuanto al flujo, según las condiciones fisiológicas pueden darse diferentes comportamientos. Sin embargo, su comportamiento principal está caracterizado por un flujo positivo en un menor periodo de tiempo, seguido de un flujo negativo originado por el cierre de la válvula aórtica, pudiendo llegar incluso a producirse un segundo impulso de sangre durante la diástole ventricular motivado por el retroceso de la sangre en la aorta descendente.

Las repercusiones que presenta la aterosclerosis sobre el comportamiento cardiovascular son principalmente dos: la pérdida de elasticidad de las arterías y el estrechamiento de la luz del vaso (estenosis). La perdida de elasticidad produce un aumento de las presiones máximas alcanzadas [10], así como la modificación del patrón de onda debido a que la reflexión de las ondas se producen con mayor rapidez. Por otro lado, la estenosis reduce el flujo de sangre.

\section{MODELO ELÉCTRICO DEL SISTEMA CARDIOVASCULAR}

Para desarrollar un modelo del SCV se ha optado por describir primero su comportamiento mediante estructuras eléctricas con un funcionamiento conceptual análogo.

A partir del modelo de Windkessel [16] y las modificaciones sugeridas en [18], el SCV puede ser analizado a través de un circuito eléctrico, como se muestra en la Figura 2 (sombreado naranja) [13]. En este modelo, la circulación sistémica y pulmonar quedan simplificadas a una resistencia periférica total $\left(\mathrm{R}_{s}\right)$ que considera la oposición de todas las arterias y venas, y un condensador que emula la elasticidad de los vasos sanguíneos, y con ello la capacidad de albergar sangre $\left(\mathrm{C}_{s}\right)$. La morfología destacada del cayado aórtico también se encuentra reflejada en el modelo a través de la resistencia $\mathrm{R}_{c}$, así como las fuerzas inerciales que experimenta el flujo debido a su carácter pulsátil (L). Por otro lado, la dinámica del corazón es reducida a la parte izquierda y las correspondientes válvulas. La capacidad de contracción del VI, recogido por la ley de Frank-Starling, es considerada como un condensador variable $(C(t))[13,15]$, mientras

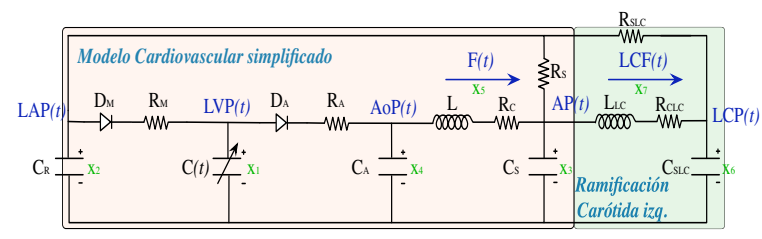

Figura 2: Modelo eléctrico del SCV (sombreado naranja) extendido a la arteria carótida común izquierda (sombreado verde).

que la aurícula izquierda es interpretada como un elemento pasivo de acumulación $\left(\mathrm{C}_{s}\right)$. En cuanto a las válvulas, son modeladas como un diodo no ideal, componente que permite identificar el control del flujo de sangre en función de la presión entre sus extremos $[13,4]$. Además, la elasticidad de la válvula aórtica es caracterizada con un condensador $\left(\mathrm{C}_{A}\right)$ que recoge la dinámica de la onda diacrótica. Respecto al carácter cíclico del SCV, es logrado a través un circuito realimentado.

En segundo lugar, al sistema anterior se le incluye el modelo de las arterias centrales, concretamente de la arteria carótida común izquierda, de acuerdo con [18, 13] (ver el sombreado verde en la Figura 2). Como se ha comentado anteriormente, se elige este lecho vascular por su elevada recurrencia en presentar placas de ateroma [10]. El modelo completo se ha establecido en base a la distribución anatómica del sistema circulatorio y los criterios empleados para desarrollar el modelo de Windkessel de 4 elementos [16], donde la resistencia $\mathrm{R}_{C L C}$ modela de la oposición al flujo y la bifurcación de dicha arteria, mientras que $\mathrm{R}_{S L C}$ modela la resistencia de retorno al corazón. El elemento $\mathrm{L}_{L C}$ simboliza la inercia experimentada en gran medida por el flujo de la arteria aorta, provocando grandes variaciones del flujo. La capacidad $\mathrm{C}_{S L C}$ representa la elasticidad.

Una vez completado el esquema eléctrico que representa el funcionamiento cardiovascular, se extraen las relaciones matemáticas entre las diferentes variables hemodinámicas, atendiendo a la definición física de los componentes que constituyen el modelo eléctrico equivalente, salvo en el caso de los diodos y el componente que emula el VI que se explican a continuación. Los diodos son descritos según la siguiente función:

$$
r(\xi)= \begin{cases}\xi, & \text { si } \xi \geq 0 \\ 0, & \text { si } \xi<0\end{cases}
$$

mientras que su comportamiento resistivo viene determinado por la resistencia en serie asociada. Por otra parte, el condensador de capacidad variable $C(t)$ refleja la elasticidad durante la contracción del VI y es definida como la inversa de la 
Tabla 1: Variables del modelo del SCV.

\begin{tabular}{lll}
\hline Variable & Abreviatura & Significado clínico (unidad) \\
\hline$x_{1}(\mathrm{t})$ & $\mathrm{LVP}(\mathrm{t})$ & Presión del ventrí́culo izquierdo $(\mathrm{mmHg})$ \\
$x_{2}(\mathrm{t})$ & $\mathrm{LAP}(\mathrm{t})$ & Presión de la aurícula izquierda $(\mathrm{mmHg})$ \\
$x_{3}(\mathrm{t})$ & $\mathrm{AP}(\mathrm{t})$ & Presión arterial $(\mathrm{mmHg})$ \\
$x_{4}(\mathrm{t})$ & $\mathrm{AoP}(\mathrm{t})$ & Presión aórtica $(\mathrm{mmHg})$ \\
$x_{5}(\mathrm{t})$ & $\mathrm{F}(\mathrm{t})$ & Flujo de sangre $(\mathrm{ml} / \mathrm{s})$ \\
$x_{6}(\mathrm{t})$ & $\mathrm{LCP}(\mathrm{t})$ & Presión arteria carótida común izquierda $(\mathrm{mmHg})$ \\
$x_{7}(\mathrm{t})$ & $\mathrm{LCF}(\mathrm{t})$ & Flujo de sangre en la arteria carótida $(\mathrm{ml} / \mathrm{s})$ \\
\hline
\end{tabular}

elastancia $E(t)=\frac{1}{C(t)}$. La elastancia es el estado de contracción, que relaciona la presión $(L V P(t))$ y volumen $(L V V(t))$ del VI de acuerdo con la siguiente expresión [13]:

$$
E(t)=\frac{L V P(t)}{L V V(t)-V_{0}},
$$

donde $V_{0}$ es el volumen de referencia, que se corresponde con el volumen ventricular teórico a una presión cero. La descripción empleada en este documento se basa en los estudios realizados en [15], donde describen la elastancia de una persona en un buen estado cardiovascular como

$$
E_{H}=\left(E_{\max }-E_{\text {min }}\right) E_{n}\left(t_{n}\right)+E_{\text {min }},
$$

siendo $E_{\max }$ y $E_{\min }$ constantes relacionadas con el volumen máximo y mínimo diástole. El parámetro $E_{n}\left(t_{n}\right)$ es la elastancia normalizada, con $t_{n}=$ $t /(0,2+0,15 \cdot 60 / H R)$, donde $H R$ es la frecuencia cardíaca. La elastancia normalizada, conocida como función de doble colina, viene dada por la siguiente ecuación:

$$
E_{n}\left(t_{n}\right)=1,55\left(\frac{\left(\frac{t_{n}}{0,7}\right)^{1,9}}{1+\left(\frac{t_{n}}{0,7}\right)^{1,9}}\right)\left(\frac{1}{1+\left(\frac{t_{n}}{1,17}\right)^{21,9}}\right)
$$

Es necesario matizar que en (3) se realiza el escalado de la elastancia normalizada entre los valores $E_{\max }$ y $E_{\text {min }}$, ya que el valor normalizado presenta la misma morfología para un corazón sano o enfermo [17]. Las afecciones cardíacas son modeladas mediante la ponderación de (3) con el factor $\delta$, con $0<\delta \leq 1$ (el valor unitario corresponde a una persona sana).

Las ecuaciones diferenciales que rigen el comportamiento del sistema se obtienen seleccionando las variables recogidas en la Tabla 1 y empleando la definición de los propios componentes, así como las leyes de tensiones y corrientes de Kirchoff.

Así, el estado $x_{1}$ es establecido a partir de la relación indicada en (2) y la relación entre el voltaje $(V)$ y la corriente $(I)$ en un condensador, es decir, $\frac{d V}{d t}=\frac{1}{C} I$, que resulta en:

$$
x_{1}=E(t)\left(L V V(t)-V_{0}\right),
$$

\begin{tabular}{|c|c|c|}
\hline Parámetro & Valor & Significado clínico \\
\hline & Resistencias & \\
\hline$R_{S}$ & $1(\mathrm{mmHg} \mathrm{s} / \mathrm{ml})$ & Resistencia periférica total. \\
\hline$R_{S L C}$ & $10(\mathrm{mmHg} \mathrm{s} / \mathrm{ml})$ & Resistencia periférica carótida común izquierda. \\
\hline$R_{M}$ & $0,005(\mathrm{mmHg} \mathrm{s} / \mathrm{ml})$ & Resistencia válvula mitral. \\
\hline & $0,001(\mathrm{mmHg} \mathrm{s} / \mathrm{ml})$ & Resistencia válvula aórtica. \\
\hline$R_{C}$ & $0,0398(\mathrm{mmHg} \mathrm{s} / \mathrm{ml})$ & Resistencia arteria aorta. \\
\hline \multirow[t]{2}{*}{$R_{C L S}$} & $0,2(\mathrm{mmHg} \mathrm{s} / \mathrm{ml})$ & Resistencia carótida común izquierda. \\
\hline & Capacidad & \\
\hline$C_{R}$ & $8,8(\mathrm{ml} / \mathrm{mmHg})$ & Elasticidad aurícula izquierda. \\
\hline$C_{S}$ & $1,33(\mathrm{ml} / \mathrm{mmHg})$ & Elasticidad sistémica. \\
\hline$C_{A}$ & $0,08(\mathrm{ml} / \mathrm{mmHg})$ & Elasticidad válvula aórtica. \\
\hline \multirow{2}{*}{$C_{L C}$} & $0,09(\mathrm{ml} / \mathrm{mmHg})$ & Elasticidad carótida común izquierda. \\
\hline & Inductancia & \\
\hline$L_{S}$ & $0,0005\left(\mathrm{mmHg} \mathrm{s}^{2} / \mathrm{ml}\right)$ & Inercia asociada a la arteria aorta. \\
\hline \multirow[t]{2}{*}{$L_{S L C}$} & $0,03\left(\mathrm{mmHg} \mathrm{s}^{2} / \mathrm{ml}\right)$ & Inercia asociada a la carótida común izquierda. \\
\hline & Ventrículo izquierdo & \\
\hline$E_{\max }$ & $2(\mathrm{mmHg} / \mathrm{ml})$ & Contractilidad máxima. \\
\hline$E_{\operatorname{mix}}$ & $0,006(\mathrm{mmHg} / \mathrm{ml})$ & Contractilidad mínima. \\
\hline$V_{0}$ & $10(\mathrm{ml})$ & Volumen del ventrículo izquierdo a presión cero. \\
\hline$H R$ & $75(\mathrm{lpm})$ & Frecuencia cardíaca. \\
\hline
\end{tabular}

donde $L V V(t)=\int\left(I_{D_{M}}-I_{D_{A}}\right) d t$, siendo $I_{D_{M}}$ e $I_{D_{A}}$ las corrientes correspondientes a los diodos
Tabla 2: Parámetros del modelo del SCV.

$D_{M}$ y $D_{A}$, respectivamente. Por tanto, la derivada del estado $x_{1}$ es

$\dot{x}_{1}=\frac{1}{C(t)}\left(-\dot{C}(t) x_{1}+\frac{1}{R_{M}} r\left(x_{2}-x_{1}\right)-\frac{1}{R_{A}} r\left(x_{1}-x_{4}\right)\right)$.

Las derivadas de los demás estados, como se muestra a continuación, son deducidas directamente a partir de las leyes mencionadas:

$$
\begin{aligned}
\dot{x}_{2}= & \frac{1}{C_{R}}\left(-\left(\frac{1}{R_{S}}+\frac{1}{R_{S L C}}\right) x_{2}+\right. \\
& \left.\frac{1}{R_{S}} x_{3}+\frac{1}{R_{S L C}} x_{6}-\frac{1}{R_{M}} r\left(x_{2}-x_{1}\right)\right) \\
\dot{x}_{3}= & \frac{1}{C_{S}}\left(\frac{1}{R_{S}} x_{2}-\frac{1}{R_{S}} x_{3}+x_{5}-x_{7}\right) \\
\dot{x}_{4}= & \frac{1}{C_{A}}\left(-x_{5}+\frac{1}{R_{A}} r\left(x_{1}-x_{4}\right)\right) \\
\dot{x}_{5}= & \frac{1}{L}\left(-x_{3}+x_{4}-R_{C} x_{5}\right) \\
\dot{x}_{6}= & \frac{1}{C_{S L C}}\left(\frac{1}{R_{S L C}} x_{2}-\frac{1}{R_{S L C}} x_{6}+x_{7}\right) \\
\dot{x}_{7}= & \frac{1}{L_{L C}}\left(x_{3}-x_{6}-R_{L C} x_{7}\right)
\end{aligned}
$$

Se define así un sistema de marcado carácter no lineal a causa de la función descrita en (1) y autónomo debido al carácter cíclico de los términos $\dot{C}(t)$ y $1 / C(t)$. De esta forma se describe la dinámica cardiovascular de manera continua.

Los valores utilizados para definir las variables del modelo son recogidos en la Tabla 2, los cuales se han extraído de [13, 18]. La magnitud de los parámetros se expresa en unidades de referencias híbridas, que permiten obtener la información en el sistema de unidades más utilizado a nivel clínico.

Con el propósito de su validación, el modelo eléctrico se ha implementado con la herramienta Simscape Electronics de Matlab/Simulink, y mediante la programación de las ecuaciones anteriores en Matlab. La dinámica cardiovascular simu- 
lada se representa en la Figura 3, donde se observan las formas de ondas hemodinámicas para un adulto con una frecuencia cardíaca de 75 latidos por minuto (lpm). Para este caso particular, las presiones arteriales sistólica y diastólica son 112 y $77 \mathrm{mmHg}$, la presión media arterial (calculada como $M A P=(S A P+2 D A P) / 3)$ es $92 \mathrm{mmHg}$, y el gasto cardíaco es de 5,90 l/min para una fracción de eyección de 78,71 ml/latido. Las presiones sistólicas y diastólicas del VI son 117 y 7 mmHg, los volúmenes telesistólicos y telediastólico son 67 y $137 \mathrm{ml}$, y la presión de la aurícula varía entre 7-12 mmHg durante el ciclo cardíaco. Estos resultados, además de los obtenidos por otros medios de verificación (omitidos por cuestiones de espacio), son consistentes con los parámetros hemodinámicos reales $[8,7,5, ?, 12]$, demostrando así que el modelo ofrece unos resultados acordes a la realidad.

Por último, la Figura 4 muestra las formas de onda de la presión y el flujo en la carótida común izquierda, donde se identifican las principales características que la definen [10]: 1) desfase de tiempo respecto a la presión arterial, 2) ligero aumento de la presión, 3) mayor acentuación de la onda diacrótica, y 4) flujo negativo y segundo impulso de sangre en diástole ventricular.

\section{MODELO HIDRÁULICO PARA ROBOTS NADADORES}

Con el objeto de trasladar el modelo eléctrico descrito a un modelo hidráulico que permita el ensayo de robots nadadores, se establecerán las equivalencias entre componentes eléctricos e hidráulicos, así como las consideraciones pertinentes que permitan adaptar la dinámica descrita a una implementación física. Como se ha visto, los componentes eléctricos utilizados han sido resistencias, condensadores e inductancias, cuyo comportamiento hidráulico puede ser modelado por cambios de sección, depósitos o tuberías de gran longitud [2],

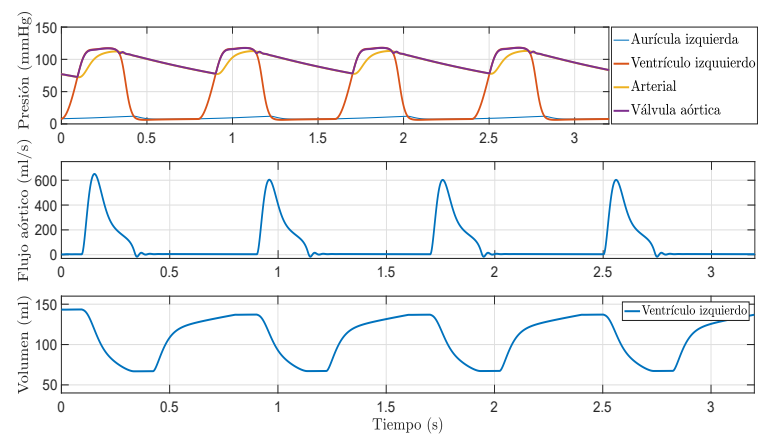

Figura 3: Resultados del modelo eléctrico.

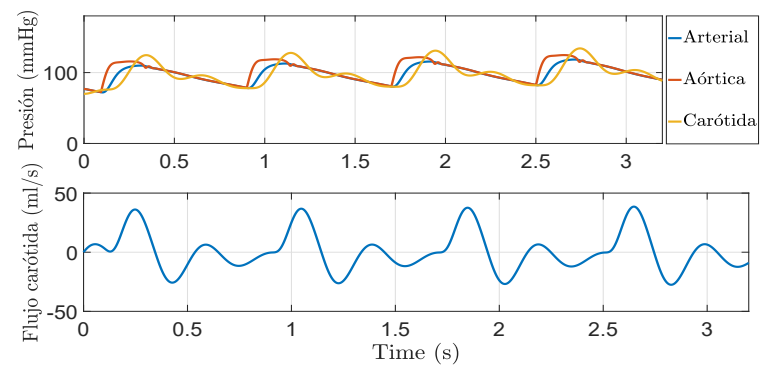

Figura 4: Resultados relativos a la arteria carótida común izquierda (a partir del modelo eléctrico).

respectivamente, como se detalla a continuación. Respecto a los diodos, son sustituidos por válvulas unidireccionales.

La resistencia hidráulica es directamente equivalente a una resistencia eléctrica y se denotarán más adelante como $R_{H_{i}}$, donde $i$ se refiere al subíndice usado en el modelo eléctrico; esta misma nomenclatura se utilizará para el resto de elementos. Un condensador en términos hidráulicos es un depósito, cuya constante de capacidad equivalente es identificada como $C_{H}=\frac{A}{\rho g}$, donde $A$ es la sección del depósito, $\rho$ es la densidad del fluido y $g$, la gravedad. Respecto a la inductancia eléctrica, es similar a la hidráulica, pudiendo expresarse de la siguiente forma $L_{H}=\frac{l \rho}{A}$, donde $l$ es la longitud de la tubería [2].

Respecto la contracción muscular del VI, el modelo eléctrico consideraba un condensador de capacidad variable para satisfacer la ley de Frank-Starling y así describir la actividad muscular del VI como (5). Para el modelo hidráulico es emplear una bomba, concretamente en este trabajo se utilizará una bomba de pistón [4], que junto con una adecuada estrategia de control, permitirá emular el funcionamiento del corazón de acuerdo con la ley indicada. Este tipo de actuador se puede interpretar como un depósito de volumen variable en función de la posición del pistón.

Analizando la dinámica de la bomba como un depósito de volumen variable, se obtiene que la variación de presión es función de los caudales de entrada, salida y la variación de volumen causada por el desplazamiento del pistón. Por lo tanto, la derivada de $x_{1}$ para el modelo hidráulico se define como:

$$
\dot{x}_{1}=\frac{1}{C_{p}}\left(Q_{i}-Q_{o}-A_{p} \nu\right),
$$

donde $C_{p}$ define la capacidad del pistón, $Q_{i}$ y $Q_{o}$ son los caudales de entrada y salida, respectivamente, $A_{p}$ es la sección del pistón (véase la Figura 5), y $\nu=\dot{x}_{p}$ se corresponde con la velocidad de desplazamiento del pistón de acuerdo al criterio de signos indicado en la figura. 


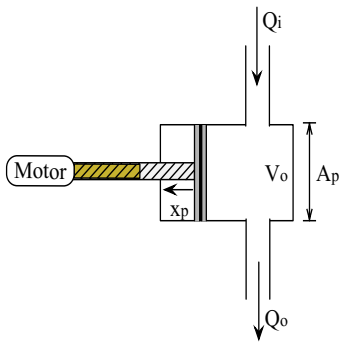

Figura 5: Esquema del VI emulado mediante una bomba de pistón.

Teniendo en cuenta las equivalencias descritas, el esquema eléctrico presentado en la Figura 2 se corresponde con el mostrado en la Figura 6. Por su parte, el modelo matemático anteriormente descrito viene dado en este caso como sigue:

$$
\begin{aligned}
\dot{x}_{1}= & \frac{1}{C_{p}}\left(\frac{1}{R_{H_{M}}} r\left(x_{2}-x_{1}\right)\right. \\
& \left.-\frac{1}{R_{H_{A}}} r\left(x_{1}-x_{4}\right)\right)-\frac{A_{p}}{C_{p}} \nu \\
\dot{x}_{2}= & \frac{1}{C_{H_{R}}}\left(-\left(\frac{1}{R_{H_{S}}}+\frac{1}{R_{H_{S L C}}}\right) x_{2}+\right. \\
& \frac{1}{R_{H_{S}}} x_{3}+\frac{1}{R_{H_{S L C}}} x_{6}- \\
& \left.\frac{1}{R_{H_{M}}} r\left(x_{2}-x_{1}\right)\right) \\
\dot{x}_{3}= & \frac{1}{C_{H_{S}}}\left(\frac{1}{R_{H_{S}}} x_{2}-\frac{1}{R_{H_{S}}} x_{3}+x_{5}-\right. \\
& \left.x_{7}\right) \\
\dot{x}_{4}= & \frac{1}{C_{H_{A}}}\left(-x_{5}+\frac{1}{R_{H_{A}}} r\left(x_{1}-x_{4}\right)\right) \\
\dot{x}_{5}= & \frac{1}{L_{H}}\left(-x_{3}+x_{4}-R_{H_{C}} x_{5}\right) \\
\dot{x}_{6}= & \frac{1}{C_{H_{S L C}}}\left(\frac{1}{R_{H_{S L C}}} x_{2}-\frac{1}{R_{H_{S L C}}} x_{6}\right. \\
& \left.+x_{7}\right) \\
\dot{x}_{7}= & \frac{1}{L_{H_{L C}}}\left(x_{3}-x_{6}-R_{H_{L C}} x_{7}\right) \\
= &
\end{aligned}
$$

donde la dinámica de $\dot{x}_{1}$ es la impuesta por la bomba de pistón, y la derivada de los demás estados se corresponde con las del modelo eléctrico a través de las equivalencias descritas. El modelo obtenido es un sistema no lineal y no autónomo

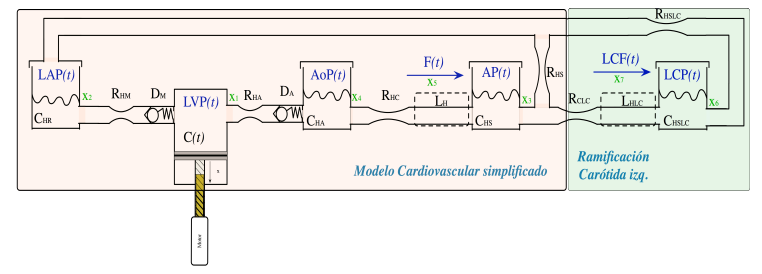

Figura 6: Modelo hidráulico del SCV. cuya entrada $\nu$ modifica la presión en la cámara del pistón $\left(x_{1}\right)$.

A diferencia del modelo eléctrico, éste no presenta un carácter autónomo como consecuencia del tipo de mecanismo escogido para implementar la contracción del VI, motivo por el cual se hace necesario aplicar una estrategia de control que regule la velocidad de desplazamiento del pistón para así emular las variaciones de presión del VI.

\subsection{Diseño del controlador}

La estrategia de control utilizada para el sistema anterior se basa en una linealización por realimentación, método que permite realizar una transformación algebraica entre la entrada y salida deseada e interpretar el sistema como lineal, y por lo tanto, aplicar cualquier técnica de control lineal, reduciendo así la complejidad del control [14].

Para linealizar la relación (14) se puede tomar la variable de entrada $\nu$ del sistema como:

$\nu=-\frac{C_{p}}{A_{p}}\left(u-\frac{1}{C_{p}}\left(\frac{r\left(x_{2}-x_{1}\right)}{R_{H_{M}}}-\frac{r\left(x_{1}-x_{4}\right)}{R_{H_{A}}}\right)\right)$,

que permite considerar una relación lineal entre la salida del sistema $\left(y=x_{1}\right)$ y la variable $u$, siendo $u$ la entrada equivalente de la dinámica linealizada, que es interpretada de orden relativo uno al obtener una relación de integración entre la salida y entrada equivalente $(\dot{y}=u)$. Considerando esta nueva relación lineal, se establece como criterio de diseño la siguiente ley de control:

$$
u=\dot{y}_{d}+\lambda e,
$$

donde $y_{d}$ es la salida deseada, $e$ es el error definido de la forma $e=y_{d}-y_{1}$ y $1 / \lambda$ es la constante de tiempo del error.

Finalmente, sustituyendo (22) en (21), se obtiene

$\nu=-\frac{C_{p}}{A_{p}}\left(\dot{y}_{d}-\frac{1}{C_{p}}\left(\frac{r\left(x_{2}-x_{1}\right)}{R_{H_{M}}}-\frac{r\left(x_{1}-x_{4}\right)}{R_{H_{A}}}\right)+\lambda e\right)$,

que permite alcanzar la referencia de acuerdo con la ley de control establecida. Es necesario destacar el valor positivo del parámetro $\lambda$ para asegurar la estabilidad del sistema, siendo mayor la velocidad del sistema cuanto mayor sea su valor.

Conviene mencionar que, en la simulación, las variables $\dot{y}_{d}$ y $y_{d}$ son proporcionadas por el modelo eléctrico, mientras que las demás son obtenidas por medición del emulador hidráulico. Es necesario destacar que mediante el modelo y estrategia de control propuesta se logra independizar la dinámica emulada de la caracterización de la contracción ventricular (ley de Frank-Starling), pudiendo utilizar datos reales como referencia. La 


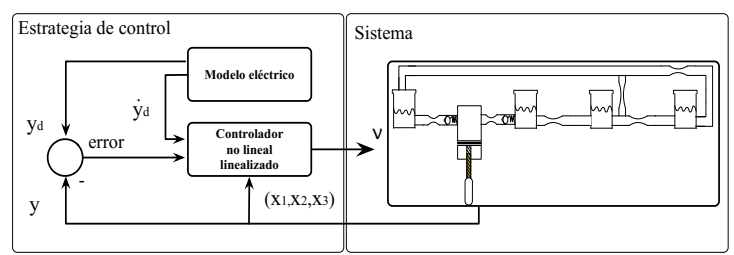

Figura 7: Diagrama de bloques de la estrategia de control para el modelo hidráulico del SCV.

Figura 7 muestra un diagrama de bloques de la estrategia de control empleada.

Para verificar la estrategia de control propuesta, así como el propio modelo desarrollado, el modelo hidráulico es implementado con la herramienta Simscape Fluids de Matlab/Simulink. Las tuberías que conectan los diferentes depósitos y válvulas se establecen con un diámetro de 2 pulgadas (diámetro suficiente para asegurar un libre movimiento de los robots nadadores), sin que éste se encuentre limitado por las restricciones de contorno. Respecto a las dimensiones de los depósitos, su sección y el valor inicial del fluido, son obtenidos a partir de las equivalencias indicadas anteriormente y los valores de la Tabla 2. Además, con el objeto de lograr escalar a nivel macroscópico los fenómenos microscópicos producidos a causa de la interacción de los microrrobots con la hemodinámica cardiovascular, el fluido a utilizar será aceite $30 \mathrm{~W}$, cuya viscosidad es 109,408 cSt y densidad $852,5 \mathrm{~kg} / \mathrm{m}^{3}$ a $35^{\circ}$, características que, para robots de pequeñas dimensiones, permiten emular las condiciones en las que se encontrarían sujetos estos robots en el sistema circulatorio humano.

La Figura 8 muestra los resultados de simulación correspondientes al modelo hidráulico con el control. Como se puede observar, éstos son similares a los obtenidos con el modelo eléctrico, por lo que este modelo se puede considerar válido para emular la dinámica del SCV. El error cuadrático medio normalizado (NRMSE) de los resultados obtenidos en la variable de control con este modelo con res-

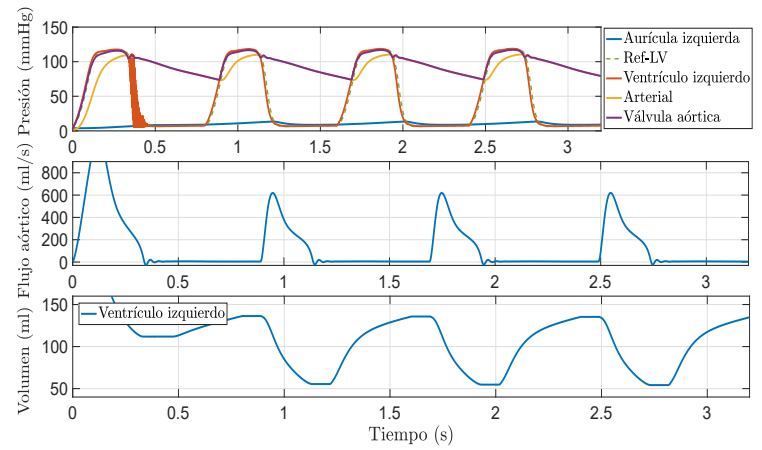

Figura 8: Resultados del modelo hidráulico.

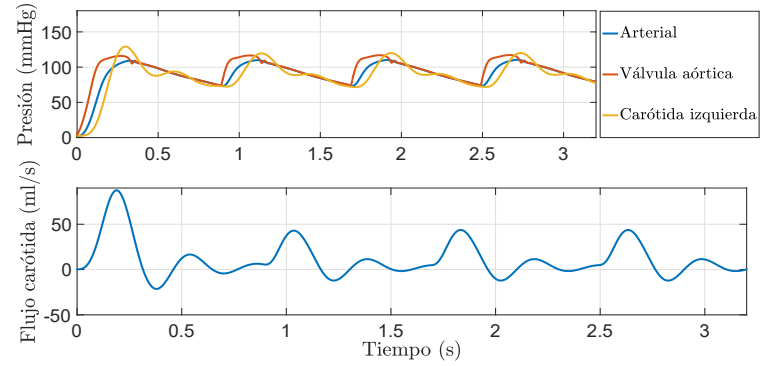

Figura 9: Resultados relativos a la arteria carótida común izquierda (modelo hidráulico).

pecto al eléctrico es de 1,90\%, que evidentemente se puede considerar despreciable y al mismo tiempo admisible considerando la variabilidad de los parámetros hemodinámicos, mientras que el desfase es de 10,50 ms.

Respecto a la presión en la carótida izquierda común y el flujo, representados en la Figura 9, también muestran el comportamiento esperado.

\section{CONCLUSIONES}

Este artículo ha presentado un modelo de simulación del sistema cardiovascular (SCV) en el entorno de Matlab/Simulink, más concretamente de la zona de mayor riesgo cardiovascular, la arteria carótida. Está basado en un modelo eléctrico que contempla la dinámica de contracción del corazón de acuerdo con la ley de Frank-Starling y su carácter cíclico y autónomo. Así mismo, se ha extendido el modelo a la dinámica de las arterias centrales, concretamente a la arteria carótida izquierda, con motivo del elevado índice de mortalidad que produce la enfermedad de arteriosclerosis en este vaso sanguíneo.

A partir de él, y haciendo una serie de equivalencias entre dominios, se ha desarrollado un modelo hidráulico del SCV que emula el comportamiento del sistema en esa zona y que, a diferencia del anterior, no presentaba carácter autónomo. Es por ello que se ha utilizado la estrategia de linealización por realimentación para diseñar el control para este modelo. Con el propósito de validarlos, se han implementado ambos modelos en el entorno Matlab/Simulink mediante la herramienta Simscape. Los resultados de simulación obtenidos, tanto con el modelo eléctrico extendido como con el hidráulico propuesto, han demostrado el correcto funcionamiento al compararlos con los recogidos en la bibliografía de ambos.

Como trabajo futuro, se plantea realizar una verificación cuantitativa del comportamiento hemodinámico de la arteria carótida común izquierda, 
el estudio de diferentes estrategias de control independientes del modelo eléctrico y la construcción física del modelo hidráulico desarrollado como plataforma de ensayo para robots nadadores tipo flagelo eucariótico y bacteriano de pequeñas dimensiones que permita emular las condiciones en las que se encontrarían estos robots navegando por el sistema circulatorio humano.

\section{Agradecimientos}

Este trabajo ha sido parcialmente financiado por Fondos FEDER (Programa Operativo FEDER de Extremadura 2014-2020), a través de la Ayuda a Grupos de la Junta de Extremadura con número de expediente GR15178, y el Ministerio de Economía y Competitividad, a través del proyecto con referencia DPI2016-80547-R.

\section{Referencias}

[1] H.R. Arabnia and Q.N. Tran. Emerging trends in applications and infrastructures for computational biology, bioinformatics, and systems biology: Systems and applications. Emerging Trends in Computer Science and Applied Computing. Elsevier Science, 2016.

[2] D. K. Chaturvedi. Modeling and simulation of systems using MATLAB and Simulink. CRC Press, 2009.

[3] L. P Dasi, H. A. Simon, P. Sucosky, and A. P. Yoganathan. Fluid mechanics of artificial heart valves. Clinical and Experimental Pharmacology and Physiology, 36(2):225-237, 2009.

[4] K. Gwak, H. D. Kim, and C. Kim. Feedback linearization control of a cardiovascular circulatory simulator. IEEE Transactions on Control Systems Technology, 23(5):19701977, 2015.

[5] Edwards Lifesciences. Normal hemodynamic parameters and laboratory values, 2017.

[6] Y. Liu, P. Allaire, Y. Wu, H. Wood, and D. Olsen. Construction of an artificial heart pump performance test system. Cardiovascular Engineering, 6(4):151-158, 2006.

[7] LiDCO Ltd. Normal hemodynamic parameters, 2017.

[8] F. Martini, J.L. Nath, and E.F. Bartholomew. Fundamentals of Anatomy \& Physiology. Benjamin-Cummings Publishing Company, 2015.
[9] B. J. Nelson, I. K. Kaliakatsos, and J. J. Abbott. Microrobots for minimally invasive medicine. Annual review of biomedical engineering, 12:55-85, 2010.

[10] W. Nichols, M. O'Rourke, and C. Vlachopoulos. McDonald's Blood Flow in Arteries, Sixth Edition: Theoretical, Experimental and Clinical Principles. CRC Press, 2011.

[11] World Health Organization. Cardiovascular diseases, 2016.

[12] H. Priebe and K. Skarvan. Cardiovascular physiology. BMJ Publishing Group, 1995.

[13] M. A. Simaan. Rotary Heart Assist Devices, pages 1409-1422. Springer Berlin Heidelberg, Berlin, Heidelberg, 2009.

[14] J.J.E. Slotine and W. Li. Applied Nonlinear Control. Prentice-Hall International Editions. Prentice-Hall, 1991.

[15] N. Stergiopulos, J. Meister, and N. Westerhof. Determinants of stroke volume and systolic and diastolic aortic pressure. American Journal of Physiology-Heart and Circulatory Physiology, 270(6):H2050-H2059, 1996.

[16] N. Westerhof, J. Lankhaar, and B. E. Westerhof. The arterial windkessel. Medical $\& 3$ biological engineering \& computing, 47(2):131141, 2009.

[17] N. Westerhof, N. Stergiopulos, and M. Noble. Snapshots of hemodynamics: an aid for clinical research and graduate education. Springer Science \& Business Media, 2010.

[18] Y. Yu, J. R. Boston, M. A Simaan, and J. Antaki. Estimation of systemic vascular bed parameters for artificial heart control. IEEE Transactions on Automatic Control, 43(6):765-778, 1998. 